\title{
CRESCIMENTO DE MUDAS DE PUPUNHEIRA (Bactris gasipaes H.B.K) SOB DIFERENTES NÍVEIS DE SALINIDADE ${ }^{1}$
}

\author{
ANTONIO RODRIGUES FERNANDES ${ }^{2}$ \\ JANICE GUEDES DE CARVALHO \\ NILTON CURI ${ }^{3}$ \\ PAULO DE TÁCITO GONTIJO GUIMARÃES ${ }^{4}$ \\ JOSÉ EDUARDO BRASIL PEREIRA PINTO 5
}

\begin{abstract}
RESUMO - A salinidade geralmente causa redução do crescimento das plantas, induzindo a desordens nutricionais causadas pelo excesso de sais, principalmente $\mathrm{NaCl}$. Com o objetivo de estudar a influência de diferentes níveis de salinidade sobre o crescimento de mudas de pupunheira (Bactris gasipaes H.B.K.), instalouse um experimento usando solução nutritiva, em condições de casa-de-vegetação, no Departamento de Ciência do Solo da Universidade Federal de Lavras. O experimento foi realizado em blocos casualizados com sete tratamentos e quatro repetições. Os tratamentos consta-
\end{abstract}

ram das seguintes doses: $0,0 \mathrm{Na}$ e $0,5 \mathrm{Cl} ; 1,0 \mathrm{Na}$ e 0,5 $\mathrm{Cl}$ (controle), e 5,0;15,0;30,0;60,0 e $120,0 \mathrm{mmol} \mathrm{L}^{-1}$ de $\mathrm{NaCl}$. A pupunheira foi beneficiada pela presença do $\mathrm{Na}$ na solução, nas dosagens de até $5 \mathrm{mmol} \mathrm{L}^{-1} \mathrm{de} \mathrm{NaCl}$; no entanto, maior crescimento de mudas foi obtido com $1 \mathrm{mmol} \mathrm{L}^{-1}$ de $\mathrm{Na}$ e $0,5 \mathrm{mmol} \mathrm{L}^{-1}$ de $\mathrm{Cl}$. A pupunheira, na fase inicial de crescimento, não se mostrou tolerante ao $\mathrm{NaCl}$ nas doses a partir de $15 \mathrm{mmol} \mathrm{L}^{-1}$ aplicadas na solução. As variáveis de crescimento correlacionaramse negativa e significativamente com a condutividade elétrica da solução.

TERMOS PARA INDEXAÇÃO: Bactris gasipaes, fitotoxidez por cloreto de sódio, condutividade elétrica.

\section{GROWTH OF PEACH PALM (Bactris gasipaes H.B.K) SEEDLINGS UNDER DIFFERENT SALINITY LEVELS}

\begin{abstract}
Soil salinity usually causes reduction of growth of crops inducing nutritional disorders caused by the excess of salts, mainly $\mathrm{NaCl}$. With the objective of studying the influence of different salinity levels upon growth of peach palm (Bactris gasipaes H.B.K.) seedlings, an experiment was established using nutrient solution, under greenhouse conditions at the Soil Science Departament of the Federal University Lavras Minas Gerais, state Brazil. The experimental design was in randomized blocks, with seven treatments and four replications. The
\end{abstract}

treatments consisted of the following levels of $\mathrm{NaCl}$ : 0.0 and $0.5 \mathrm{Cl} ; 1$ and $0.5 \mathrm{Cl}$ (control); $5.0 ; 15.0 ; 30.0$; 60.0 and $120.0 \mathrm{mmol} \mathrm{L}^{-1}$. The peach palm seedlings were benefited by $\mathrm{Na}$ in solution, up to $5 \mathrm{mmol} \mathrm{L}^{-1}$ of $\mathrm{NaCl}$, however higher growth was obtained with $1 \mathrm{mmol} \mathrm{L}^{-1}$ of $\mathrm{Na}$ and $0,05 \mathrm{mmol} \mathrm{L}^{-1}$ of $\mathrm{Cl}$. At the initial growth phase, the peach palm was not tolerant to $\mathrm{NaCl}$ at doses from $15 \mathrm{mmol} \mathrm{L}^{-1}$ applied in the solution. The growth variables were negatively correlated with the electric conductivity of the solution.

INDEX TERMS: Bactris gasipaes, chloride sodium phytotoxicity, electric conductivity.

1. Parte da tese de Doutorado apresentada pelo primeiro autor ao Curso de Pós-Graduação em Solos e Nutrição de Plantas na UNIVERSIDADE FEDERAL DE LAVRAS/UFLA, Caixa Postal 37 - 37200-000 - Lavras, MG.

2. Engenheiro Agrônomo, Dr., Professor Adjunto, Departamento de Ciência do Solo da Faculdade de Ciências Agrárias do Pará. Caixa Postal 917 - 66077-550 - Belém,PA. arfernan@ufpa.br.

3. Engenheiro Agrônomo, Dr., Professor Titular, Departamento de Ciência do Solo da UFLA. Bolsistas CNPq. janicegc@ufla.br; niltcuri@ufla.br.

4. Engenheiro Agrônomo, Dr., Pesquisador da EPAMIG, Lavras. paulotgg@ufla.br

5. Engenheiro Agrônomo, PhD, Professor Titular, Departamento de Agricultura da UFLA. jeduardo@ufla.br 


\section{INTRODUÇÃO}

A pupunheira (Bactris gasipaes H.B.K), em razão do seu elevado potencial produtivo, está difundida nas diferentes regiões do Brasil. No entanto, na Região Norte, onde tem grande importância como fonte alimentícia pelo alto valor nutritivo dos seus frutos, tornou-se, também, uma alternativa economicamente viável para produção de palmito.

Além de ser ecologicamente desejável o cultivo dessa espécie para produção de palmito, em substituição ao corte predatório de espécies de palmeiras espontâneas do gênero Euterpe (E. oleracea Mart. e E. edulis Mart.) em matas tropicais, essa planta apresenta algumas características agronômicas importantes, como precocidade, produtividade, adaptabilidade e capacidade de perfilhamento ao longo dos anos, além de produzir palmito de boa qualidade.

A expansão das áreas de cultivo, até mesmo para o semi-árido, tem sido acompanhada de uma melhoria do nível tecnológico, com a utilização de calagens, adubações e irrigações, o que tem possibilitado uma exploração mais precoce. No entanto, isso leva à necessidade adicional de conhecimento e ao desenvolvimento de tecnologias agronômicas que conduzam ao aumento dos cultivos viáveis economicamente e de forma sustentável.

O uso de elevadas quantidades de fertilizantes, a elevação do lençol freático ou o uso na irrigação de água salina têm sido as maiores causas da salinização dos solos (Sonnoveld \& Welles, 1988). É um problema que cresce ano a ano devido à escassez de chuvas e à demanda sempre crescente de água e fertilizantes pela agricultura moderna. Além disso, o aumento da população mundial e, conseqüentemente, da urbanização têm forçado os produtores a utilizarem cada vez mais solos marginais, inclusive aqueles salinos. Esses fatores têm aumentado a necessidade do desenvolvimento de tecnologias alternativas de uso desses solos, bem como a busca de espécies potencialmente promissoras para essas áreas.

O estresse salino provoca a redução do crescimento das plantas em razão dos desequilíbrios nutricionais na absorção e transporte de nutrientes (Yahya, 1998; Ferreira et al., 2001) e da diminuição da área foliar (Munns, 1983). Sintomas de fitotoxidez, seja por sódio $(\mathrm{Na})$ ou por cloro $(\mathrm{Cl})$ nas espécies cultiva- das, também são atribuídos ao desbalanço nutricional (Kuiper et al., 1990; Lima, 1997); por outro lado, sintomas de deficiência por $\mathrm{Na}$, principalmente, são raros, visto que esse elemento é considerado como benéfico, ou seja, essencial apenas para algumas espécies ou sob certas condições (Marschner, 1995).

As espécies da família Arecaceae têm apresentado características favoráveis, tendo em vista que o seu crescimento tem sido estimulado e a sua produção aumentada pela presença do $\mathrm{Na}$ (Bonneau et al., 1993; Magat et al., 1993), e que quantidades elevadas de $\mathrm{Cl}$ têm sido requeridas por tais espécies para expressar todo o seu potencial produtivo (Braconnier \& D'Auzac, 1990; Ollagnier, 1985; Uexkull, 1985; Marschner, 1995; Sobral \& Leal, 1999), inclusive a pupunheira (Carvalho et al., 1996). No entanto, não se conhecem as concentrações adequadas de $\mathrm{Cl}$ e de $\mathrm{Na}$, na solução, que possam estimular o crescimento e promover maior equilíbrio nutricional da pupunheira, bem como a sua tolerância à salinidade. Na pupunheira, não são conhecidos sintomas de deficiência de $\mathrm{Na}$ ou de fitotoxidez por excesso de cloreto de sódio. Assim sendo, com este trabalho objetivou-se estudar a influência de diferentes doses de cloreto de sódio $(\mathrm{NaCl})$, em solução nutritiva, sobre o crescimento em mudas de pupunheira.

\section{MATERIAL E MÉTODOS}

O experimento foi conduzido em casa-devegetação do Departamento de Ciência do Solo da Universidade Federal de Lavras - UFLA (Lavras - MG), situada a $918 \mathrm{~m}$ de altitude, $21^{\circ} 14^{\prime} \mathrm{S}$ de latitude e $45^{\circ}$ 00'W de longitude.

Adotou-se o delineamento em blocos casualizados, com sete tratamentos e quatro repetições. Os tratamentos constaram dos seguintes níveis: $0,0 \mathrm{Na}$ e $0,5 \mathrm{Cl}$; $1,0 \mathrm{Na}$ e $0,5 \mathrm{Cl} \mathrm{mmol} \mathrm{L}{ }^{-1}$ (controle); e 5,0; 15,0; 30,0; 60,0 e $120,0 \mathrm{mmol} \mathrm{L}^{-1}$ de $\mathrm{NaCl}$, correspondendo aos seguintes valores de condutividades elétricas (CE): 1,28; 1,$34 ; 2,06 ; 2,88 ; 4,42 ; 7,51$ e $13,20 \mathrm{dS} \mathrm{m}^{-1}$. O tratamento-controle foi definido a partir da solução nutritiva determinada por Dufour et al., (1978) para o dendezeiro. Determinou-se a $\mathrm{CE}$ a $25^{\circ} \mathrm{C}$, com condutivímetro Micronal 330, visando a fundamentar a tolerância da pupunheira à salinidade, tendo em vista que os limites de tolerância são expressos em dS m${ }^{-1}$ e as referências en-

Ciênc. agrotec., Lavras. V.27, n.2, p.278-284, mar./abr., 2003 
contram-se normalmente nesse padrão. Esses limites foram relacionados com as doses de $\mathrm{NaCl}$, ajustando-se a equação: $\mathrm{CE}=1,3973+0,0992 \mathrm{NaCl}, \mathrm{R}^{2}=0,999$.

As unidades experimentais foram constituídas por um vaso contendo uma planta com uma solução nutritiva básica para macronutrientes composta de: $\mathrm{NO}_{3}=8 ; \mathrm{NH}_{4}=2 ; \mathrm{P}=1 ; \mathrm{K}=2 ; \mathrm{Ca}=2 ; \mathrm{Mg}=1,5 ; \mathrm{S}$ $=1 ; \mathrm{Na}=1,0$ e $\mathrm{Cl}=0,5 \mathrm{mmol} \mathrm{L}{ }^{-1}$. Os sais que forneceram os nutrientes foram: $\mathrm{Ca}\left(\mathrm{NO}_{3}\right)_{2} ; \mathrm{NH}_{4} \mathrm{NO}_{3} ; \mathrm{Na}-$ $\mathrm{NO}_{3} ; \mathrm{Mg}\left(\mathrm{NO}_{3}\right)_{2} ; \mathrm{KH}_{2} \mathrm{PO}_{4} ; \mathrm{K}_{2} \mathrm{SO}_{4}$ e $\mathrm{MgCl}_{2}$. As quantidades de $\mathrm{Na}$ ou $\mathrm{Cl}$ utilizadas nos sais para o preparo da solução foram diminuídas das doses de $\mathrm{NaCl}$. As concentrações de micronutrientes e seus respectivos sais foram: $\mathrm{B}=0,20\left(\mathrm{H}_{3} \mathrm{BO}_{4}\right) ; \mathrm{Cu}=0,05(\mathrm{Cu}-$ $\left.\mathrm{SO}_{4} \cdot 5 \mathrm{H}_{2} \mathrm{O}\right) ; \mathrm{Fe}=3,00($ FeEDTA $) ; \mathrm{Mn}=0,35(\mathrm{Mn}-$ $\left.\mathrm{SO}_{4} \cdot \mathrm{H}_{2} \mathrm{O}\right) ; \mathrm{Mo}=0,02\left(\left(\mathrm{NH}_{4}\right)_{6} \mathrm{Mo}_{7} \mathrm{O}_{27} \cdot 4 \mathrm{H}_{2} \mathrm{O}\right)$ e $\mathrm{Zn}=$ $0,05\left(\mathrm{ZnSO}_{4} \cdot 7 \mathrm{H}_{2} \mathrm{O}\right) \mathrm{mg} \mathrm{L}^{-1}$.

As sementes de pupunheira foram colocadas para germinar em bandejas plásticas contendo areia. Após 60 dias da germinação, as plântulas foram selecionadas e colocadas em bandejas coletivas com capacidade para $36 \mathrm{~L}$ de solução nutritiva, a $1 / 4$ da força iônica durante 30 dias e a $1 / 2$ da força iônica durante mais 30 dias. Após esse período, as plantas foram selecionadas e transferidas para vasos com $3 \mathrm{~L}$ de solução com força iônica total. Depois de 30 dias em tais recipientes, as plantas foram distribuídas uniformemente em blocos e a solução modificada, conforme os tratamentos, permanecendo assim por quatro meses. As soluções foram renovadas a cada 15 dias nos dois primeiros meses e semanalmente a partir do terceiro mês. Após esse período, foram transferidas para vasos com capacidade para $9 \mathrm{~L}$, permanecendo, dessa maneira, por mais dois meses, perfazendo um período experimental de seis meses. Nesses recipientes, as soluções foram renovadas a cada 20 dias no primeiro mês e a partir daí, a cada 15 dias.

A solução nutritiva foi mantida sob aeração constante durante todo o período experimental, e o seu volume foi mantido constante pela reposição diária de água desionizada, ao passo que o pH da solução de cultivo foi apenas monitorado.

Por ocasião da colheita do experimento, foram medidos a altura do coleto ao ápice da última folha completamente formada e o perímetro do coleto das plantas. Depois de colhido, o material vegetal foi sepa- rado em raiz, estipe e folha, e, em seguida, foi medida fotometricamente a área foliar. Posteriormente, todo o material vegetal foi lavado em água destilada corrente e seco em estufa com circulação de ar a $65-70^{\circ} \mathrm{C}$, até peso constante. A matéria seca correspondente a cada uma das partes da planta foi pesada, moída e armazenada em frascos de vidro, para as determinações químicas.

No extrato obtido por digestão nitroperclórica do material vegetal, foram obtidos os teores de $\mathrm{P}$ por colorimetria, de $\mathrm{K}$ e $\mathrm{Na}$ por fotometria de chama, de $\mathrm{S}$ por turbidimetria e de $\mathrm{Ca}$ e $\mathrm{Mg}$ por espectrofotometria de absorção atômica. $\mathrm{O}$ teor de $\mathrm{N}$ total foi determinado pelo método semimicro Kjeldahl, enquanto o teor de $\mathrm{Cl}$ foi determinado por titulação com $\mathrm{Ag}\left(\mathrm{NO}_{3}\right)_{2}$. Todos os nutrientes determinados seguiram metodologia descrita por Malavolta et al., (1997).

Os resultados foram submetidos à análise de variância e de regressão utilizando o sistema de análises estatísticas SANEST (Zonta \& Machado, 1991). Testaram-se diversos modelos para as equações, sendo a escolha baseada no coeficiente de determinação e na sua significância. Foram determinadas correlações lineares entre as variáveis de crescimento.

\section{RESULTADOS E DISCUSSÃO}

\section{Sintomas visuais de fitotoxidez}

Sintomas de deficiência de Na, no tratamento em que esse elemento foi omitido, foram caracterizados apenas pela redução do crescimento do sistema radicular e da parte aérea, enquanto sintomas de fitotoxidez por $\mathrm{NaCl}$, nas doses a partir de $15 \mathrm{mmol} \mathrm{L}{ }^{-1}$, foram caracterizados por reduções no número de folhas e sinais de injúrias, como clorose, seguida de necrose nas pontas e margens das folhas. A redução no número de folhas, bem como as injúrias, tornaram-se mais severas à medida que foi aumentada a concentração salina da solução.

Os sintomas de fitotoxidez são atribuídos a desbalanços nutricionais no citoplasma (Lima, 1997), a uma redução da translocação de citocininas para as folhas (Kuiper et al., 1990), com conseqüente elevação do conteúdo de ácido absísico e ao acúmulo de sais no ápice e nas margens das folhas, onde a trans- 
piração é maior, principalmente de cloreto (Richards, 1974).

\section{Avaliação biométrica}

Os valores médios de altura, perímetro do coleto, área foliar, matéria seca das folhas, estipes e raízes das plantas encontram-se na Figura 1. A análise de regressão entre tais variáveis e a salinidade apresentou um comportamento similar, caracterizado por um decréscimo consistente e significativo com o aumento do estresse salino, tomando como referência o tratamento com 1 de $\mathrm{Na}$ e 0,5 de $\mathrm{Cl} \mathrm{mmol} \mathrm{L}^{-1}$ (controle). Redução e/ou inibição do crescimento das plantas pela salinidade tem sido atribuída ao efeito osmótico, associado à toxicidade pela absorção excessiva dos íons $\mathrm{Na}$ e $\mathrm{Cl}$ e ao desequilíbrio nutricional causado pelos distúrbios na absorção e/ou distribuição dos nutrientes essenciais (Yahya, 1998).

A altura e o perímetro foram as variáveis biométricas menos afetadas pelo estresse salino (Figura 1). No entanto, as reduções de tais medidas atingiram $50 \%$ e $54 \%$, respectivamente, em relação ao tratamentocontrole. Para a área foliar e a matéria seca das folhas, estipes, raízes, parte aérea e total, as reduções foram bastante expressivas, cujos valores corresponderam a 73, 63, 81, 89, 73 e 78\%, respectivamente (Figura 1). Reduções significativas da matéria seca da parte aérea de $79 \%$ e das raízes de $55 \%$ de mudas de goiabeira foram constatadas por Ferreira et al. (2001), aos 50 dias sob estresse salino de $150 \mathrm{mmol} \mathrm{L}^{-1}$ de $\mathrm{NaCl}$.

$\mathrm{O}$ efeito mais comum da salinidade é sobre o crescimento devido à redução da área foliar que afeta a assimilação de carbono pela planta que, por sua vez, conduz a uma menor taxa fotossintética (Munns, 1993). Por outro lado, a redução da área foliar pode aumentar, indiretamente, a concentração de solutos na folha, contribuindo para o ajustamento osmótico, ou seja, para a adaptação da planta à salinidade, a menos que os solutos elevem-se a níveis tóxicos em compartimentos celulares específicos da folha (Läuchli \& Epstein, 1990).
O sistema radicular da pupunheira foi a parte da planta mais afetada pela salinidade, diferentemente do que ocorre na maioria das espécies, em que a parte aérea é mais sensível ao estresse salino do que a raiz, devido aos desequilíbrios entre os cátions, em consequiência das complexas interações no sistema de transporte (Munns, 1993). Entretanto, a relação raiz/parte aérea aumentou, em relação ao tratamentocontrole, na ausência do $\mathrm{Na}$ e na dose de $5 \mathrm{mmol} \mathrm{L}^{-1}$ de $\mathrm{NaCl}$, para depois decrescer a partir da dose de 15 mmol $\mathrm{L}^{-1}$. Tais diferenças de crescimento nas partes das plantas sob diferentes níveis de salinidade podem estar relacionadas à adaptação ao novo ambiente. Aumentos da relação raiz/parte aérea em resposta à salinidade foram também constatados por Yang et al. (1990) em Sorghum bicolor Moench. e S. halepense (L.) Pers.. Por outro lado, Storey (1995), em citrus, e Ferreira et al. (2001), em goiabeira, constataram maior crescimento da parte aérea em relação às raízes, em resposta à salinidade. A pupunheira beneficiou-se da presença do $\mathrm{Na}$ em baixas concentrações na solução, tendo em vista que o tratamento-controle proporcionou o maior incremento em todas as variáveis biométricas estudadas. Por outro lado, o tratamento zero de $\mathrm{Na}$ afetou mais o crescimento do que aquele representado por $5 \mathrm{mmol} \mathrm{L}{ }^{-1}$ de $\mathrm{NaCl}$.

O estudo de correlação entre as variáveis de crescimento (Quadro 1) foi realizado para avaliar as interrelações entre as mesmas perante a resposta da pupunheira à salinidade. Verificou-se que existe uma correlação positiva e significativa entre as variáveis de crescimento como resultado do estresse salino. De maneira oposta, tais variáveis correlacionaram-se negativa e significativamente com a CE. Isso revela a sensibilidade da pupunheira à salinidade, representada pela redução das variáveis de crescimento. Estudos de correlação entre as características de crescimento, de rendimento e da CE da solução foram utilizados por Satti et al. (1995) para demonstrar a resposta do tomate à salinidade. 

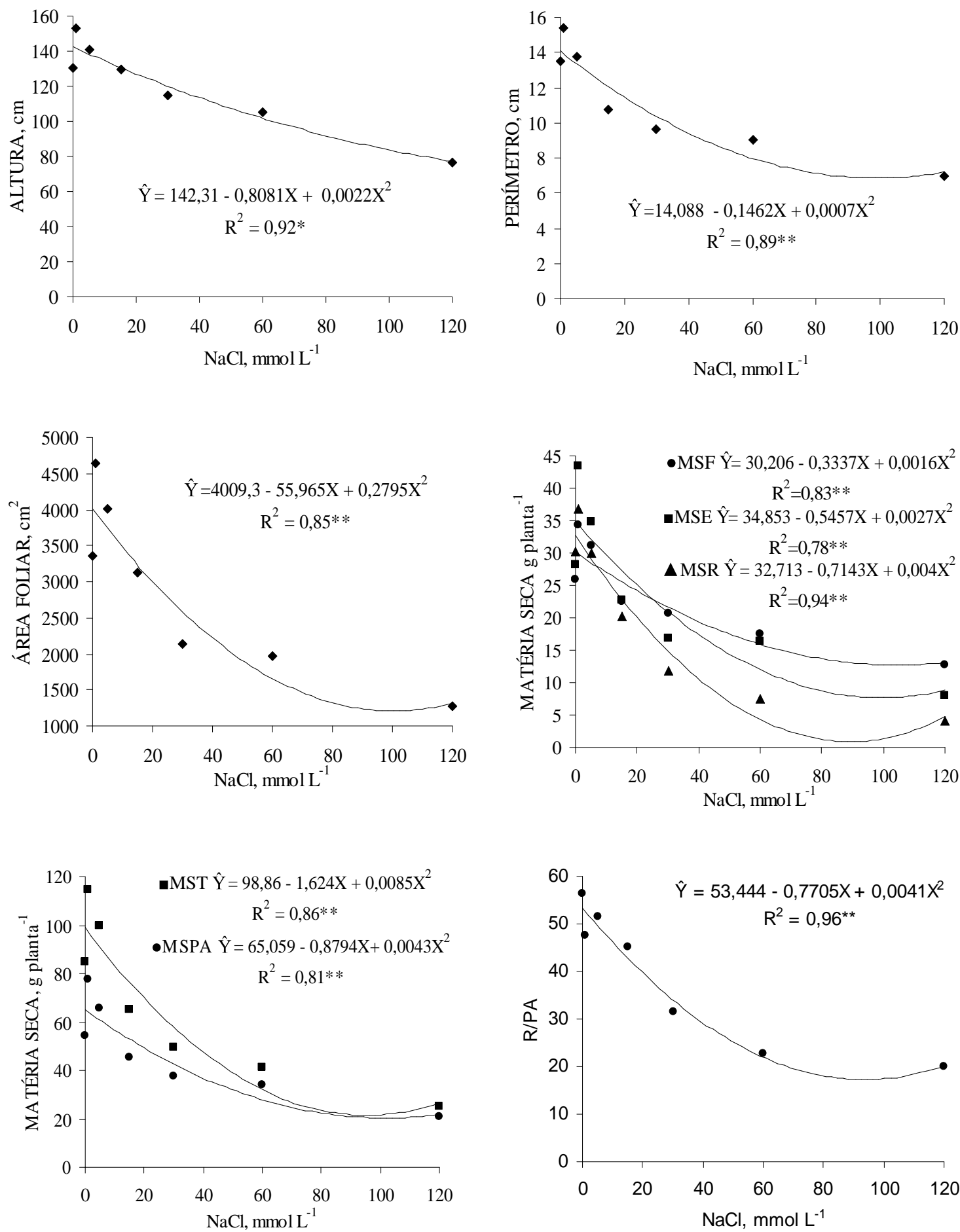

FIGURA 1 - Altura, perímetro do coleto, área foliar, matéria seca de folhas (MSF), de estipes (MSE), de raízes (MSR), da parte aérea (MSPA) e total (MST) e relação raiz/parte aérea de mudas de pupunheira, em função das doses de $\mathrm{NaCl} .(*),(* *)$ significativos a 5 e $1 \%$ pelo teste $\mathrm{F}$, respectivamente.

Ciênc. agrotec., Lavras. V.27, n.2, p.278-284, mar./abr., 2003 
QUADRO 1 - Correlações entre as variáveis de crescimento altura (ALT), perímetro do coleto (PER), área foliar (AF), matéria seca de folhas (MSF), estipe (MSE), raízes (MSR), parte aérea (MSPA) e total (MST), relação raiz/parte aérea e condutividade elétrica em $\mathrm{d} S \mathrm{~m}^{-1}$ (CE) da solução em resposta aos níveis de salinidade.

\begin{tabular}{cccccccccc}
\hline Variáveis & ALT & PER & AF & MSF & MSE & MSR & MSPA & MST & R/PA \\
\hline PER & $0,87^{* *}$ & - & - & - & - & - & - & - & \\
AF & $0,93^{* *}$ & $0,91^{* *}$ & - & - & - & - & - & - & \\
MSF & $0,91^{* *}$ & $0,90^{* *}$ & $0,95^{* *}$ & - & - & - & - & - & \\
MSE & $0,91^{* *}$ & $0,92^{* *}$ & $0,91^{* *}$ & $0,88^{* *}$ & - & - & - & - & \\
MSR & $0,87^{* *}$ & $0,93^{* *}$ & $0,90^{* *}$ & $0,86^{* *}$ & $0,94^{* *}$ & - & - & - & \\
MSPA & $0,93^{* *}$ & $0,94^{* *}$ & $0,96^{* *}$ & $0,96^{* *}$ & $0,98^{* *}$ & $0,93^{* *}$ & - & - & \\
MST & $0,92^{* *}$ & $0,95^{* *}$ & $0,95^{* *}$ & $0,94^{* *}$ & $0,98^{* *}$ & $0,97^{* *}$ & $0,99^{* *}$ & - & \\
R/PA & $0,68^{* *}$ & $0,74^{* *}$ & $0,66^{* *}$ & $0,57^{*}$ & $0,71^{* *}$ & $0,87^{* *}$ & $0,67^{* *}$ & $0,76^{* *}$ & \\
CE & $-0,95^{* *}$ & $-0,89^{* *}$ & $-0,87^{* *}$ & $-0,87^{* *}$ & $-0,83^{* *}$ & $-0,88^{* *}$ & $-0,86^{* *}$ & $-0,85^{* *}$ & $-0,91^{* *}$ \\
\hline
\end{tabular}

$(*),(* *)$ significativo a 5 e $1 \%$ pelo teste $\mathbf{F}$, respectivamente.

\section{CONCLUSÕES}

A pupunheira foi beneficiada pela presença do $\mathrm{Na}$ na solução, nas dosagens de até $5 \mathrm{mmol} \mathrm{L}^{-1} \mathrm{de} \mathrm{NaCl}$; no entanto, maior crescimento de mudas foi obtido com $1 \mathrm{mmol} \mathrm{L}^{-1}$ de $\mathrm{Na}$ e $0,5 \mathrm{mmol} \mathrm{L}^{-1}$ de $\mathrm{Cl}$.

A pupunheira, na fase inicial de crescimento, não se mostrou tolerante ao $\mathrm{NaCl}$ nas doses a partir de 15 mmol L $\mathrm{L}^{-1}$ aplicadas na solução.

As variáveis de crescimento correlacionaram-se negativa e significativamente com a condutividade elétrica da solução.

\section{REFERÊNCIAS BIBLIOGRÁFICAS}

BONNEAU, X.; OCHS, R.; KITU, W. T.; YUSWOHADI. Chlorine: an essential element in the mineral nutrition of hybrid coconuts in Lampung (Indonesia). Oleagineux, Paris, v. 48, n. 4, p. 179-190, 1993.

BRACONNIER, S.; D'AUZAC, J. Chloride and stomatal conductance in coconut. Oléagineux, Paris, v. 45 , n. 6, p. 259-266, 1990.

CARVALHO, J. G.; MATOS, A. O.; GUERRERO, J. A.; VARGAS, J. A. Efeito da relação $\mathrm{Cl}$ :S no teor de macronutrientes em pupunha cultivada em areia e solução nutritiva. In: REUNIÃO BRASILEIRA DE FERTILIDADE DO SOLO E NUTRIÇÃO DE
PLANTAS, 22., 1996, Manaus. Resumos expandidos... Manaus: SBCS, 1996. p. 654-655.

DUFOUR, F.; QUENCEZ, P.; SCHMITY, G. Technique de culture en solutions nutritives du palmier à huile et du cocotier. Oléagineux, Paris, v. 33, n. 10, p. 485490, Oct. 1978.

FERREIRA, R. G.; TÁVORA, F. J. A. T.; HERNANDEZ, F. F. F. Distribuição da matéria seca e composição química das raízes, caule e folhas de goiabeira submetida a estresse salino. Pesquisa Agropecuária Brasileira, Brasília, v. 36, n. 1, p. 79-88, jan. 2001.

KUIPER, D.; SCHUIT, J.; KUIPER, P. J. C. Actual cytokinin concentrations in plant tissue as na indicator for salts resistence in cereals. Plant and Soil, Dordrecht, v. 123, n. 2, p. 243-250, Apr. 1990.

LÄUCHLI, A.; EPSTEIN, E. Plant response to saline and sodic conditions. In: TANJI, K. K. Agricultural salinity assessment and management. New York: ASCE, 1990. Cap. 6, p. 113-137.

LIMA, L. A. Efeitos de sais no solo e na planta. In: CONGRESSO BRASILEIRO DE ENGENHARIA AGRÍCOLA, 26., 1997, Campina Grande. Manejo e controle da salinidade na agricultura irrigada. Campina Grande: UFPB/SBEA, 1997. cap. 4, p. 112-136. 
MAGAT, S. S.; PADRONES, G. D.; ALFORJA, L. M. Residual effects of three chloride fertilizers on yield and leaf nutrient levels of coconuts grown on an inland soil of Davao (Mindanao, Philippines). Oleagineux, Paris, v. 48 , p. $237-242,1993$.

MALAVOLTA, E.; VITTI, G. C.; OLIVEIRA, S. A. Avaliação do estado nutricional das plantas: princípios e aplicações. 2. ed. Piracicaba: POTAFOS, 1997. 319 p.

MARSCHNER, H. Mineral nutrition of higher plant. 2. ed. New York: Academy Press, 1995. 889 p.

MUNNS, R. Physiological processes limiting plant growth in saline soils: some dogmas and hypotheses. Plant, Cell and Environment, Oxford, v. 16, p. 15-24, 1993.

OLLAGNIER, M. Ionic reactions and fertilizer management in relation to drought resistance of perennial oil crops (oil palm and coconut). Oleagineux, Paris, v. 40, p. 1-10, 1985.

RICHARDS, L. A. Diagnostico y rehabilitación de suelos salinos y sódicos. 6. ed. Mexico: Limusa, 1974. $160 \mathrm{p}$.

SATTI, S. M. F.; LOPEZ, M.; AL-RAWAHY, S. A. Effects of saline nutrient solutions on the growth and accumulation of mineral elements in some tomato cultivars. Communications in Soil Science and Plant Analysis, Montecelo, v. 26, p. 2097-2106, 1995.
SOBRAL, L. F; LEAL, M. L. S. Resposta do coqueiro à adubação com uréia, superfosfato simples e cloreto de potássio em dois solos do Nordeste do Brasil. Revista Brasileira de Ciência do Solo, Campinas, v. 23, p. 8589, 1999.

SONNOVELD, C.; WELLES, G. W. H. Yield and quality of rockwool-grown tomatoes as affected by various in EC-value and climatic conditions. Plant and Soil, Dordrecht, v. 111, p. 37-42, 1988.

STOREY, R. Salt tolerance, ion relations and the effect of root medium on the response of citrus to salinity. Plant Physiology, Rockville, v. 22, p. 1-14, 1995.

UEXKULL, H. R. von. Chlorine in the nutrition of palm trees. Oléagineux, Paris, v. 40, p. 7-74, 1985.

YAHYA, A. Salinity effects on growth and on uptake and distribution of sodium and some essential mineral nutrients in sesame. Journal of Plant Nutrition, New York, v. 21, n. 7, p. 1439-1451, 1998.

YANG, Y. W.; NEWTON, J. R.; MILLER, F. R. Salinity tolerance in sorghum whole plant response. Crop Science, Madison, v. 30, p. 775-781, 1990.

ZONTA, E. P.; MACHADO, A. A. Sistema de análise estatística para microcomputadores (SANEST). Pelotas: UFPel - Departamento de Matemática e Estatística, 1991. $101 \mathrm{p}$. 